\title{
20. Chronic pain in patients with COVID-19: Cross sectional study
}

Andreas Liampas ${ }^{1, *}$, Christianna Ioannou ${ }^{1}$, Artemios Artemiadis $^{1}$, Georgios Hadjigeorgiou $^{1}$, Panagiotis Zis ${ }^{1}$

${ }^{1}$ Medical School, University of Cyprus, Nicosia, Cyprus. * liampasand@gmail.com

Background: Coronavirus disease 2019 (COVID-19) is a contagious disease caused by SARS-CoV-2. Symptoms of COVID-19 are variable, but often include fever, cough, headache, fatigue, breathing difficulties, and loss of smell or taste. Neurological complications include cerebrovascular incidents, acute polyneuropathy and myelitis. The aim of this crosssectional study was to establish the prevalence of chronic pain in COVID-19 patients.

Methods: All participants were prospectively evaluated. Pain was assessed with the use of the painDETECT and the DN4 questionnaires.

Results: Fifty-two COVID019 patients (67\% females, mean age $48.4 \pm 16.1$ years) and 52 age and gender matched healthy volunteers were recruited. Patients were evaluated on average $4.2 \pm 2.9$ months after being diagnosed with COVID-19. From the classic acute COVID-19 symptoms, 50\% had anosmia/hyposmia, 48\% ageusia/hypogeusia, 48\% fatigue, $40 \%$ cough, 39\% headache, $35 \%$ myalgias and $31 \%$ fever. The prevalence of chronic pain was $70 \%$ in the COVID- 19 group and $73 \%$ in the healthy volunteer group. The most commonly reported painful area in both groups was lower back (35\% in the COVID-19 group and 31\% in the healthy control group). In 7 patients chronic pain (6 neuropathic, 1 nociceptive) developed after COVID-19 whereas 3 patients reported worsening of their pre-existing chronic pain (2 neuropathic, 1 nociceptive). In the COVID-19 group, patients with pain were significantly older compared to patients without (51.8 \pm 17.0 years vs $40.9 \pm 10.7$ years, $p=0.022$ ). No differences between the two sub-groups were found regarding gender, BMI, COVID-19 symptoms, COVID-19 symptoms duration or interval since COVID-19 infection.

Conclusions: Chronic pain is very prevalent in patients suffered from COVID-19 and its prevalence is similar to the prevalence observed in the general population. However, one in 5 COVID-19 patients reports that chronic pain developed or deteriorated after their infection. Chronic pain, particularly neuropathic, should be considered as a symptom of the postCOVID syndrome. 